\title{
Processes of hypernuclei formation in relativistic ion collisions
}

\author{
Alexander Botvina ${ }^{1,2}$ and Marcus Bleicher ${ }^{1}$ \\ ${ }^{1}$ Frankfurt Institute for Advanced Studies, J.W.Goethe University, Frankfurt am Main, Germany \\ ${ }^{2}$ Institute for Nuclear Research, Russian Academy of Sciences, Moscow, Russia
}

\begin{abstract}
The study of hypernuclei in relativistic ion collisions open new opportunities for nuclear and particle physics. The main processes leading to the production of hypernuclei in these reactions are the disintegration of large excited hyper-residues (target- and projectile-like), and the coalescence of hyperons with other baryons into light clusters. We use the transport, coalescence and statistical models to describe the whole reaction, and demonstrate the effectiveness of this approach: These reactions lead to the abundant production of multi-strange nuclei and new hypernuclear states. A broad distribution of predicted hypernuclei in masses and isospin allows for investigating properties of exotic hypernuclei, as well as the hypermatter both at high and low temperatures. There is a saturation of the hypernuclei production at high energies, therefore, the optimal way to pursue this experimental research is to use the accelerator facilities of intermediate energies, like FAIR (Darmstadt) and NICA (Dubna).
\end{abstract}

\section{Introduction}

Hypernuclei are formed when hyperons $(\Lambda, \Sigma, \Xi, \Omega)$ produced in high-energy interactions are captured by nuclei. Presently, the hypernuclear physics is still focused on spectroscopic information and is dominated by a quite limited set of reactions induced by hadrons and leptons [1,2]. However, many experimental collaborations (e.g., STAR at RHIC [3]; ALICE at LHC [4]; HypHI at GSI [5], FOPI/CBM [6], and Super-FRS/NUSTAR at FAIR [7]; BM@N and MPD at NICA [8]) have started or plan to investigate hypernuclei and their properties in reactions induced by relativistic nucleons and ions. The limits in isospin space, particle unstable states, multiple strange nuclei and precision lifetime measurements are unique topics of these fragmentation reactions. Generally, the investigation of hypernuclei is one of the rapidly progressing fields of nuclear physics, as they provide complementary methods to improve traditional nuclear studies and open new horizons for studying particle physics and nuclear astrophysics.

\section{Modelling production of hypernuclei}

For description of the hypernuclei production both dynamical and statistical approaches can be employed. We use the Ultrarelativistic Quantum Molecular Dynamics (UrQMD) model, the Hadron String Model (HSD), the Dubna Cascade Model (DCM) as the transport approaches to simulate the initial dynamical reaction stage $[9,10]$. Nucleons from overlapping parts of the projectile and target 

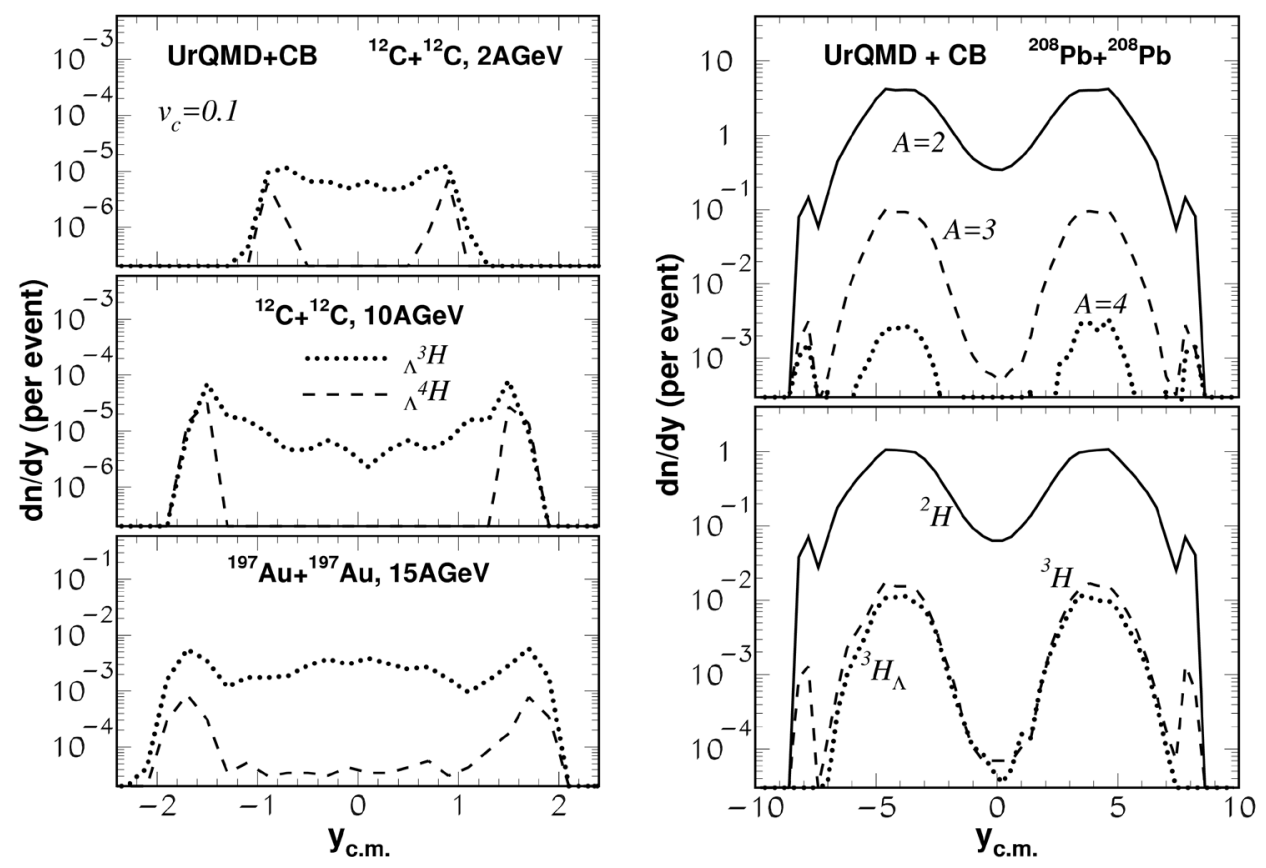

Figure 1. UrQMD and coalescence $(\mathrm{CB})$ calculations of the light nuclei rapidity distributions. Left panels are from Ref. [10]: Relative yields (per inelastic event) of ${ }_{\Lambda}^{3} \mathrm{H}$ and ${ }_{\Lambda}^{4} \mathrm{H}$ hypernuclei in carbon on carbon and gold on gold collisions at intermediate energies shown in the panels. Right panels are from Ref. [18]: Relative yields of nuclei with mass numbers $A=2,3,4$ (top) and ${ }^{2} \mathrm{H},{ }^{3} \mathrm{H},{ }_{\Lambda}^{3} \mathrm{H}$ (bottom) in lead on lead collisions at LHC energies $\left(\sqrt{s}_{N N}=2.76 \mathrm{~A} \mathrm{TeV}\right)$. Notations for the lines are in the panels.

(participant zone) interact strongly with each other and with hadrons produced in primary and secondary collisions. Nucleons from non-overlapping parts do not interact intensively, and they form residual nuclear systems, which we call spectator residues. The production of hyperons is associated with nucleon-nucleon collisions, e.g., $p+n \rightarrow n+\Lambda+K^{+}$, or collisions of secondary mesons with nucleons, e.g., $\pi^{+}+n \rightarrow \Lambda+K^{+}$. In the following the strange particles can re-scatter and undergo secondary interactions. As a result the produced hyperons populate the whole momentum space around the colliding nuclei, including the vicinity of nuclear spectators, and can be captured by the nuclear residues. This can be described both as the capture in a nuclear potential [9] and within a generalized coalescence model [10]. The coalescent mechanism can be applied for the formation of light clusters too. In such a way it is possible to describe the formation of fragments of all sizes, from lightest nuclei to heavy residues, including hyper-fragments, at all rapidities, on the event-by-event basis. The disintegration of excited hyper-fragments can be described by the generalized statistical evaporation and multifragmentation models (for big fragments) [11,12] and by the Fermi break-up model (for small fragments) [13], which were applied with great success for description of the secondary decays 
in normal reactions, see e.g. [15-17]. As a new theoretical result we have extended the elaborated previously hybrid description of nuclear fragmentation in hadron and ion relativistic collisions for hyper-nuclear matter. Within these models one can predict the production of all kind of hypernuclei (including multi-strange and the exotic ones) which are not always possible to obtain in traditional hyper-nuclear studies $[1,2]$.
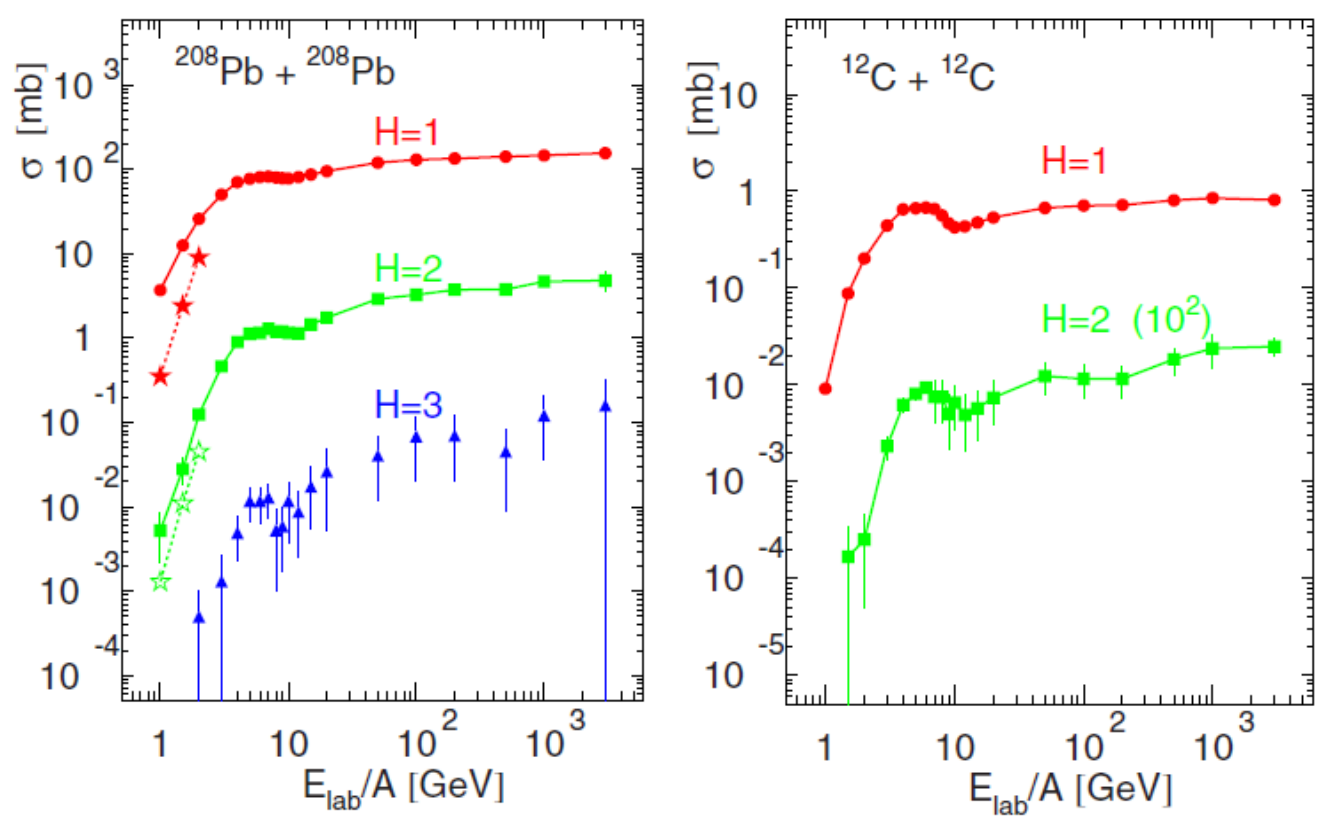

Figure 2. UrQMD (dashed lines, star symbols) and DCM (solid lines, circles, squares, triangle symbols) transport calculations: Absolute yields (millibarn) of spectator hyper-residues with capture of $H=1,2,3 \Lambda$-hyperons versus the laboratory beam energy in lead on lead (left panel) and carbon on carbon (right panel) collisions [14].

\section{Yields of hypernuclei}

Within our approach we demonstrate in Fig. 1 the rapidity distributions of light hypernuclei produced at intermediate- and very high-energy collisions. The calculations were performed overall impact parameters (minimal bias). One can see that light hypernuclei $\left({ }_{\Lambda}^{3} \mathrm{H}\right)$ can be formed at all rapidities by the coalescence mechanism. However, the production of more heavy nuclear species $\left(\right.$ e.g., $\left.{ }_{\Lambda}^{4} \mathrm{H}\right)$ is clearly shifted toward target and projectile rapidities. This is true for both intermediate (left panels) and high (right panels) energies. Moreover, at high energies, besides the expected fragmentation peaks around the target/projectile residues, we have found wide peaks related to so-called the fragmentation regions outside the midrapidity. These regions are produced because of the secondary interactions of 
strange and normal particles, which effectively decrease the particle's kinetic energy and make their capture in big clusters more probable. A chance to form an exotic state is much larger in such a case.

To evaluate the production of big hypernuclei we have calculated the formation of hyper-nuclear residues in the relativistic collisions with DCM and UrQMD (plus coalescence) models. Fig. 2 demonstrates their yields as function of the incident energy for different numbers of captured $\Lambda$ hyperons $(H)$. One can see a fast increase of the yields around the threshold energy and then a saturation up to very high energies. The cross-sections are sufficiently large even for multi-strange residues, by taking into account that modern experiments can measure nanobarn yields. The experimental vertex technique [3-5] can be effectively used. We should consider that these big residues are mostly excited and decay afterwards during the time around $10^{2}-10^{3} \mathrm{fm} / \mathrm{c}$. It was demonstrated previously that the disintegration of such excited hyper-nuclear matter leads to a very broad isotope distribution of final hypernuclei $[11,12]$. Novel multi-hyperon nuclear states can be formed in this case too.

\section{Conclusion and perspectives}

Our results demonstrate that the investigation of hypernuclei in relativistic collisions is very promising. In particular, the yields of strange spectator residues and hypernuclei are about $\sim 1-100 \mathrm{mb}$ (depending on the colliding nuclei) that is quite a lot in comparison with the traditional hypernuclear methods. An additional advantage is that very exotic hypernuclei (neutron-rich and neutron-poor ones) as well as multi-strange nuclei can be produced. The last ones are specially important for understanding the hyperon-hyperon interactions and the nature of compact astrophysical objects (e.g., neutron stars).

A.S. Botvina acknowledges the support of BMBF (Germany).

\section{References}

[1] H. Bando, T. Mottle, and J. Zofka, Int. J. Mod. Phys. A5, 4021 (1990).

[2] O. Hashimoto, H. Tamura, Prog. Part. Nucl. Phys. 57, 564 (2006).

[3] The STAR collaboration, Science 328, 58 (2010).

[4] B. Dönigus et al. (ALICE collaboration), Nucl. Phys. A904-905, 547c (2013).

[5] T.R. Saito et al. (HypHI collaboration), Nucl. Phys. A 881, 218 (2012).

[6] I. Vassiliev et al. (CBM collaboration). JPS Conf. Proc. 17, 092001 (2017).

[7] https://indico.gsi.de/event/superfrs3

[8] Topical issue NICA White Paper, Edt. D.Blaschke et al., Eur. Phys. J. A52, 211-267 (2016)

[9] A.S. Botvina et al., Phys. Rev. C 84, 064904 (2011)

[10] A.S. Botvina , J. Steinheimer, E. Bratkovskaya et al., Phys. Lett. B 742, 7 (2015)

[11] A.S. Botvina and J. Pochodzalla, Phys. Rev. C 76, 024909 (2007)

[12] A.S.Botvina et al., Phys. Rev. C94, 054615 (2016)

[13] A.S. Botvina, K.K. Gudima, J. Pochodzalla, Phys. Rev. C 88, 054605 (2013)

[14] A.S. Botvina et al., Phys. Rev. C95, 014902 (2017)

[15] H. Xi et al., Z. Phys. A 359, 397 (1997)

[16] R.P. Scharenberg et al., Phys. Rev. C 64, 054602 (2001)

[17] R. Ogul et al., Phys. Rev. C 83, 024608 (2011)

[18] A.S. Botvina, J. Steinheimer, M. Bleicher. Phys. Rev. C96, 014913 (2017) 